\title{
A DIFFERENTIAL EQUATION THEORETIC INTERPRETATION OF A GEOMETRIC RESULT OF HARTOGS
}

\author{
TAKAHIRO KAWAI
}

\begin{abstract}
A result of Hartogs on the location of singularities of holomorphic functions can be neatly proved by the theory of overdetermined systems of linear differential equations.
\end{abstract}

The pioneering work of Bochner [1] and its substantial generalization by Ehrenpreis [2] tell us that the Hartogs phenomenon concerning removable singularities of holomorphic functions of several variables are most neatly handled by the theory of overdetermined systems of linear differential equations with constant coefficients. The results of Ehrenpreis [2] and Ehrenpreis [3, Chapter XI, §2] were extended by Kawai [6] to the general situation where the equations are with variable coefficients and the boundary in question is not necessarily a linear variety. The purpose of this note is to notice that a result in Kawai [6] gives a neat proof of another interesting result (the theorem below) of Hartogs on the location of the singularities of a holomorphic function of several complex variables. (See Hartogs [4] for the original statement of the result. See also Hitotumatu [5, Chapter 3, §5]. The original result is more precise than ours in that Hartogs does not assume any regularity of the exceptional set $E$ in the theorem below.) The author hopes that the interpretation of the Hartogs theorem in the light of the theory of general overdetermined systems as is given here will help us to acquire better understanding of the nature of a domain of existence for a system of linear differential equations, a challenging and intriguing problem posed by Ehrenpreis [3, p. 338].

TheOREM (HARTOGS [4]). Let $\Omega$ be an open subset of $\mathbf{C}^{n}(n \geqslant 1)$ and let $\varphi(z, \bar{z})$ be $a$ (complex-valued) real analytic function defined on $\Omega$. Let $E$ denote $\{(z, w) \in \Omega \times \mathbf{C}$; $w=\varphi(z, \bar{z})\}$. Suppose that $\Omega \times \mathbf{C}-E$ is locally a domain of holomorphy near $E$, that is, suppose that for each point $p$ in $E$ we can find an open neighborhood $U$ of $p$ and a holomorphic function $h(z, w)$ defined on $U-E$ such that $h$ cannot be extended across E. Then $\varphi(z, \bar{z})$ is holomorphic.

Received by the editors September 5, 1985.

1980 Mathematics Subject Classification (1985 Revision). Primary 35N10; Secondary 32D05, 35N05, $46 \mathrm{~F} 15$.

Key words and phrases. Removable singularities, domain of holomorphy, system of linear differential equations, characteristic variety, characteristic. 
Proof. Let $s$ and $x_{j}$ (resp., $t$ and $y_{j}$ ) denote the real part (resp., the imaginary part) of $w$ and $z_{j}(j=1, \ldots, n)$, respectively. Let $f(x, y)$ and $g(x, y)$ respectively denote the real part and the imaginary part of $\varphi(z, \bar{z})$. Let us now regard $\Omega \times \mathbf{C}$ (resp., $E)$ as a $2(n+1)$-dimensional (resp., $2 n$-dimensional) real analytic manifold, and we denote by $X$ (resp., $Y$ ) its complexification. Let $\mathscr{M}$ denote the CauchyRiemann system on $\Omega \times \mathbf{C}$, that is,

$$
\mathscr{M}:\left\{\begin{array}{l}
\frac{1}{2}\left(\partial / \partial x_{j}+\sqrt{-1} \partial / \partial y_{j}\right) u=0 \quad(j=1, \ldots, n), \\
\frac{1}{2}(\partial / \partial s+\sqrt{-1} \partial / \partial t) u=0 .
\end{array}\right.
$$

We may, of course, regard $\mathscr{M}$ as a system of linear differential equations defined on $X$, and we denote by $V$ its characteristic variety, a subvariety of $T^{*} X$.

Since a holomorphic function on $\Omega \times C-E$ is by definition a (hyperfunction) solution $u$ of (1) defined on $\Omega \times C-E$, Theorem 1 of Kawai [6] implies that $E$ should be characteristic with respect to $\mathscr{M}$; otherwise $u$ should be extended across $E$. It then follows from the definition of the characteristicness (SatoKawai-Kashiwara [7, Chapter II, Definition 3.5.4]) that the conormal bundle $T_{Y}^{*} X$ has nontrivial intersection with $V$ over $E$. This means, by definition, that for each point $p=(q, \varphi(q))$ in $E$ we can find complex numbers $(\alpha, \beta)(\neq(0,0))$ such that

$$
\begin{aligned}
& \left(\xi_{1}, \ldots, \boldsymbol{\xi}_{n}, \eta_{1}, \ldots, \eta_{n} ; \sigma, \tau\right) \\
& \underset{\text { def }}{\alpha} \alpha\left(-\frac{\partial f}{\partial x_{1}}(q), \ldots,-\frac{\partial f}{\partial x_{n}}(q),-\frac{\partial f}{\partial y_{1}}(q), \ldots,-\frac{\partial f}{\partial y_{n}}(q) ; 1,0\right) \\
& \quad+\beta\left(-\frac{\partial g}{\partial x_{1}}(q), \ldots,-\frac{\partial g}{\partial x_{n}}(q),-\frac{\partial g}{\partial y_{1}}(q), \ldots,-\frac{\partial g}{\partial y_{n}}(q) ; 0,1\right)
\end{aligned}
$$

belongs to

$$
\begin{aligned}
V=\left\{(x, y, s, t ; \xi, \eta, \sigma, \tau) \in T^{*} X ; \xi_{j}+\sqrt{-1} \eta_{j}=0\right. & (j=1, \ldots, n) \\
& \text { and } \sigma+\sqrt{-1} \tau=0\} .
\end{aligned}
$$

Since $(\alpha, \beta) \neq(0,0)$, we may suppose $\alpha \neq 0$ and then, by the homogeneity, we may further assume $\alpha=1$. (If $\beta \neq 0$, we may normalize $\beta$ to be 1.) After this normalization we find

$$
1+\sqrt{-1} \beta=0
$$

that is, $\beta=\sqrt{-1}$. Hence we have

$$
\begin{aligned}
\left(-\frac{\partial f}{\partial x_{j}}(q)-\sqrt{-1} \frac{\partial g}{\partial x_{j}}(q)\right)+\sqrt{-1}\left(-\frac{\partial f}{\partial y_{j}}(q)-\sqrt{-1} \frac{\partial g}{\partial y_{j}}(q)\right) & =0 \\
& (j=1, \ldots, n),
\end{aligned}
$$

that is,

$$
\left(\frac{\partial}{\partial x_{j}}+\sqrt{-1} \frac{\partial}{\partial y_{j}}\right)(f+\sqrt{-1} g)=0 \quad(j=1, \ldots, n)
$$

holds identically. This means that $\varphi=f+\sqrt{-1} g$ is holomorphic, completing the proof of the theorem. 
REMARK. If the (real) codimension of the exceptional set $E$ in the theorem is equal to or greater than 4 , it is always a removable singularity for holomorphic functions. Hence the situation considered in the theorem is the only meaningful one in our context.

ACKNOwleDgments. The author would like to thank Professor T. Ohsawa for a stimulating discussion with him.

\section{REFERENCES}

1. S. Bochner, Partial differential equations and analytic continuations, Proc. Nat. Acad. Sci. U.S.A. 38 (1952), 227-230.

2. L. Ehrenpreis, A new proof and an extension of Hartogs' theorem, Bull. Amer. Math. Soc. 67 (1961) 507-509.

3. __ Fourier analysis in several complex variables, Wiley-Interscience, New York, London, Sydney, Toronto, 1970.

4. F. Hartogs, Über die aus den singulären Stellen einer analytischen Funktion mehrerer Veränderlichen bestehenden Gebilde, Acta Math. 32 (1909), 57-79.

5. S. Hitotumatu, The theory of analytic functions of several complex variables, Baifukan, Tokyo, 1960. (Japanese)

6. T. Kawai, Extension of solutions of systems of linear differential equations, Publ. Res. Inst. Math. Sci. Kyoto Univ. 12 (1976), 215-227.

7. M. Sato, T. Kawai, and M. Kashiwara, Microfunctions and pseudo-differential equations, Lecture Notes in Math., vol. 287, Springer-Verlag, Berlin, New York, Heidelberg, 1973, pp. 265-529.

Research Institute for Mathematical Sciences, Kyoto University, Kyoto 606, JaPan 